\title{
Chemical Modification of Halloysite Nanotubes for the Preparation of Nanocomposites on Non Polar Matrix
}

\author{
Alan Ramírez Guevara, Jaime Bonilla Ríos and Laura Eugenia Romero Robles \\ Department of Chemistry and Nanotechnology, Monterrey Institute of Technology and Higher Education, Monterrey 64849, N.L., \\ México
}

\begin{abstract}
In this study, we demonstrated the effects of chemical treatments for Halloysite nanotubes (HNTs) under acid and alkaline conditions using sulfuric acid and sodium hydroxide. XRD results indicate that alkaline treatment destroyed the crystalline structure and morphology for HNTs because the XRD spectrum shows the typical peaks for montmorillonite. For the acid treatment using $\mathrm{H}_{2} \mathrm{SO}_{4}$, XRD spectrum indicates an intensity reduction for the peak (001) showing a lower concentration of aluminium in the structure. Diffuse reflectance analysis shows a reduction of 40 and $15 \%$ for reflectance with $\mathrm{H}_{2} \mathrm{SO}_{4}$ and $\mathrm{NaOH}$ treatments respectively. A terephthalic acid adsorption test was realized with the HNTs, modified halloysites ( $\left.\mathrm{HNT}-\mathrm{H}_{2} \mathrm{SO}_{4}\right)$ and (HNT-NaOH) samples with a kinetic study

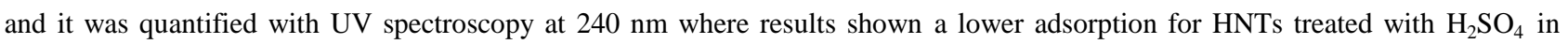
comparison with alkaline treatment and not treated HNT. A decrease of $58 \% \pm 0.3$ was achieved with the sulfuric acid treatment with not crystalline structure modification using ICP technique to quantify the sample compositions.
\end{abstract}

Keywords: Halloysite nanotubes, acid/basic treatment, aluminium sulfate, physical adsorption, Fourier-transform infrared spectroscopy (FTIR), XRD, diffuse transmittance.

\section{Introduction}

The carbonated drinks packaging industry has focused its attention on some nanoparticles such as halloysites (HNTs) to use them as additives in the nanocomposites synthesis, this due to its high availability, low cost, easy processing and a diffusion distance decrease of gas molecules, providing excellent barrier properties. The above is of great importance since it allows to reduce the $\mathrm{CO}_{2}$ permeability in polymers. HNTs have been widely used in recent years in polymer reinforcement applications [1]. An example of their performance improvement was published by Hashemifard et al. who with a $0.5 \%$ halloysites load he has obtained a $27 \%$ $\mathrm{CO}_{2}$ permeability reduction and an $8 \%$ increase in $\mathrm{CO}_{2} / \mathrm{CH}_{4}$ selectivity in a compatible polyetherimide matrix [2].

Corresponding author: Alan Ramírez Guevara, B.Sc. in chemistry sciences, research fields: materials science and industrial engineering.
HNTs form polymeric nanocomposites with a large number of advantages as a low permeability and improved mechanical properties. However, dispersion and compatibility between ionic additives such as HNTs with non-polar matrices such as PET shall be need optimized. Reason why chemical modification in halloysites surface is necessary for a better compatibility acquisition. Nowadays, the most implemented method to improve the compatibility between HNTs with polymers is the chemical surface modification to increase the HNTs affinity with non polar polymers. The modification methods selection has focused mainly on improving nanotubes physical dispersion [3].

The halloysites addition to a PET matrix for packaging uses also could introduce physical property problems such as nanocomposite transparency reduction. Fujii et al. in 2014 published a halloysite treatment with sulfuric acid to study the PVA polymer optical transmittance using untreated HNTs and HNTs 
treated with sulfuric acid [4]. The previous study achieved an optical transmittance reduction from 76 to $74 \%$, where transparency in the packaging industry is a valuable asset. However, the last study has many opportunity areas since it does not explain the structural modification for HNTs and the composition change, so we consider it important to go into depth with these details in this study.

An important case of gas permeability reduction using HNTs in a polymer matrix was developed by Monsiváis-Barrón, achieving a 66\% reduction in oxygen permeability with polypropylene, with only a $3 \%$ weight load of halloysites [5]. The previous work had been one of the reasons that inspired this work to similarly achieve a significant reduction in the permeability of $\mathrm{CO}_{2}$ in a PET matrix.

The purpose of the present work was to study the interactions of halloysite with terephthalic acid finding an economical functionalization without the use of silanization agents as well as the morphological modification of the halloysites to make them less opaque and decrease the aluminum composition through the use of economic reagents in the chemical industry such as sulfuric acid and sodium hydroxide.

\section{Materials and Reagents}

Sigma Aldrich halloysite nanotubes, Sigma Aldrich terephthalic acid, sulfuric acid, sodium hydroxide.

Equipment: Siemens D5000 diffractometer operated at $35 \mathrm{kV}$ and $25 \mathrm{~mA}$ with $\mathrm{Cu} \mathrm{K \alpha}$ radiation $(\lambda=1.5406$ $\AA$ ), from $5^{\circ}$ to $85^{\circ}$ (angle $2 \theta$ ). Optical properties such as UV absorbance and diffuse reflectance were analyzed using a spectrophotometer (Nicolet Evolution 300 PC). The FTIR Spectrum One brand (Perkin Elmer) was used for the infrared analysis by Fourier transform.

\section{Methodology}

\subsection{Treatment with Sulfuric Acid}

To achieve the morphological modification of the HNTs in sulfuric acid, 4 grams of HNTs were placed in $40 \mathrm{~mL}$ of water and the suspension was taken to ultrasound for 30 minutes. Subsequently, $10 \mathrm{~mL}$ of concentrated sulfuric acid was carefully added and maintained in magnetic stirring for 30 minutes. The present treated HNTs were filtered under vacuum and were dried in a flask for 1 hour at $100{ }^{\circ} \mathrm{C}$. Once dried they were characterized with Fourier-transform infrared spectroscopy(FTIR).

\subsection{Treatment with Sodium Hydroxide}

A treatment was carried out for the HNTs in a basic medium. To do this, the same procedure was followed as in the previous process where 4 grams of halloysite dissolved in $40 \mathrm{~mL}$ of water were placed under agitation by ultrasound for half an hour. Once the halloisites were suspended, $9 \mathrm{~g}$ of $\mathrm{NaOH}$ was added and they were stirred on a magnetic grid for half an hour. The HNTs were filtered under vacuum and placed in a heating flask for half an hour. The product of the reaction was characterized with FTIR.

\subsection{Adsorption Tests for Terephthalic Acid in Halloysite}

After the elaboration of the curve, an adsorption kinetics of terephthalic acid was performed where the adsorption of terephthalic acid of the halloysite was quantified, as well as the halloysites modified with sulfuric acid and sodium hydroxide at a $\mathrm{pH}$ of 3, 6 and 9 by an adsorption kinetics by UV spectroscopy at 240 $\mathrm{nm}$. This experiment was realized in order to analyze the affinity between modified HNTs with polar organic molecules.

\section{Results}

\subsection{Treatment with Sodium Hydroxide and Sulfuric Acid}

In Fig. 1 as you can see the peaks 2,924 and 2,500 $\mathrm{cm}^{-1}$ correspond to the aluminum sulfate compound for the FTIR spectrum of HNT treated with $\mathrm{H}_{2} \mathrm{SO}_{4}$. After the reaction the compound was washed with distilled water for later to check with FTIR analysis the disappearance of the characteristic peaks of the aluminum sulfate. With the present treatment a near decrease was achieved of $50 \%$ (measured withinductively 


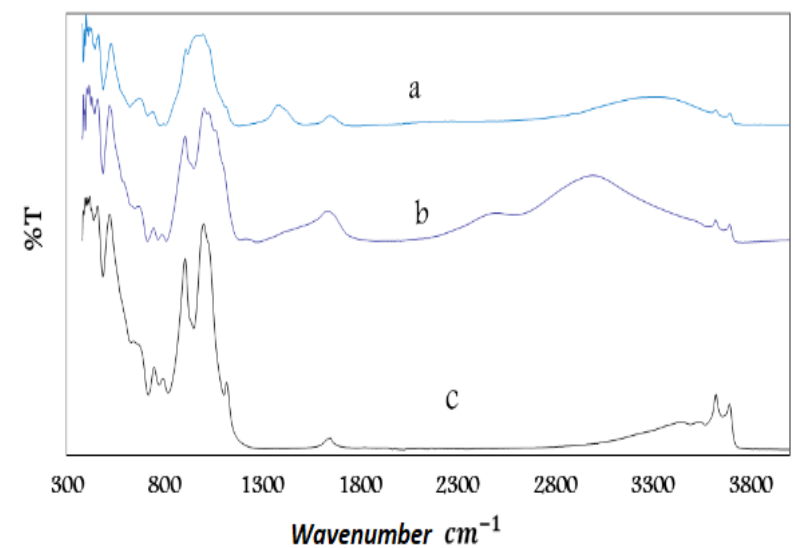

Fig. 1 FTIR spectrum for halloysite: (a)halloysite treated with sodium hydroxide and (b)treated with $\mathrm{H}_{2} \mathrm{SO}_{4}$.

coupled plasma(ICP)) in aluminum whichbrings about an increase in the interfacial area and with this the possibility that in the extrusion process the penetration of some chains of the molten polymer inside the nanoparticle.

\subsection{X-ray Diffraction (XRD)}

For halloysite treated with sodium hydroxide and sulfuric acid, XRD characterization was also performed.We can observe the XRD spectra of HNTs, halloysite treated with sulfuric acid, as well as HNTs treated with sodium hydroxide inFig. 2. Observe the drop in the peak intensity corresponding to the plane (001) in the position of $10^{\circ}$ (2 theta), contributing to the decrease of aluminum in the HNT structures treated with sodium hydroxide and sulfuric acid. In the spectrum diffraction for treatment with $\mathrm{NaOH}$ can be noted in the range of 25-35 (2 theta) a loss in crystallinity. In the range of 35-40 the structure type montmorillonite which are sheets and not nanotubes because of the above it can be mentioned that besides losing the crystalline structure very likely morphology is lost.

\subsection{Diffuse Reflectance}

In order to quantify the change in optical propertiesfor our modifications, we also carried out the diffuse reflectance characterization technique to see the increase in the reflectivity of the halloysites

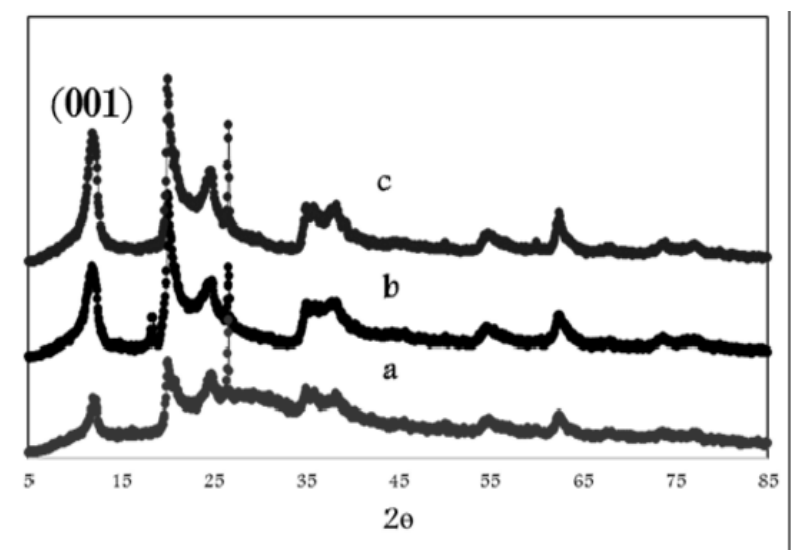

Fig. 2 The XRD spectra of halloysite: (c)halloysite treated with $\mathrm{H}_{2} \mathrm{SO}_{4}$, as well as(a) halloysite treated with $\mathrm{NaOHand}$ (b)free halloysite of treatmentare illustrated.

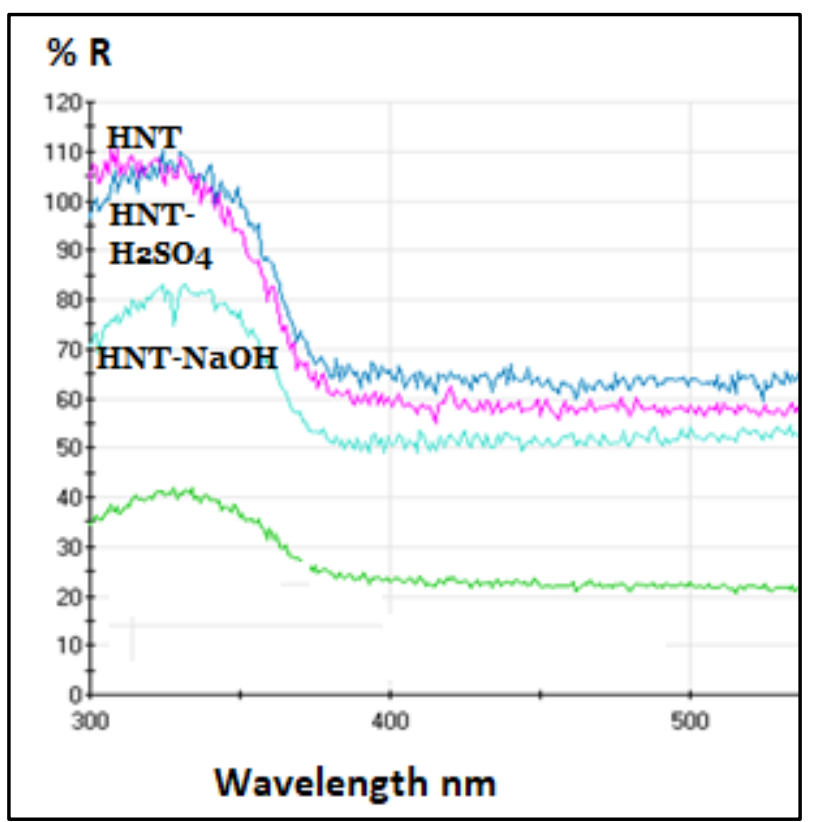

Fig. 3 In the diagram of diffuse reflectance the reflectance corresponding to haloisite can be observed without treatment and as it is radically reduced when using concentrated sulfuric acid treatment for 1 hour, treatment with 1 molar sulfuric acid for 1 hour and treated haloisite with sodium hydroxide.

treatedHNT- $\mathrm{H}_{2} \mathrm{SO}_{4}$ and $\mathrm{HNT}-\mathrm{NaOH}$, which in principle should decrease if we increase porosity of the same halloysite as we can see inFig. 3 .

The treatment with sodium hydroxide resulted, as well as the sulfuric acid treatment turned out to bemore effective in decreasing the diffuse reflectance of the halloysites without modifying their crystalline structure. This makes them more suitable for food 


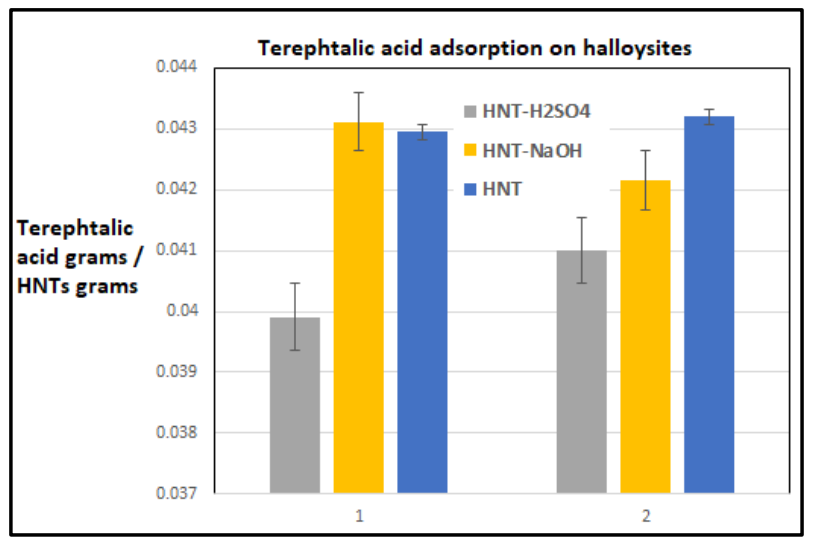

Fig. 4 This graph shows the grams of terephthalic acid absorbed by one gram of halloysite in solution.

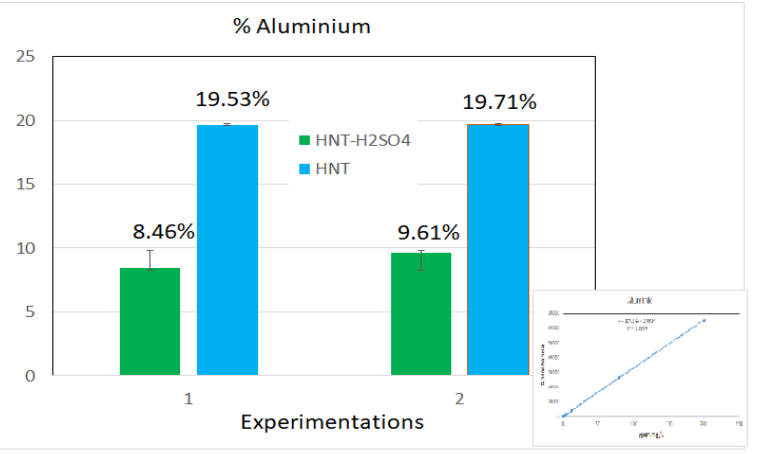

Fig. 5 In this graph is shown the \% aluminium composition for HNTs and $\mathrm{HNT}-\mathrm{H}_{2} \mathrm{SO}_{4}$. The correlation curve for aluminium was 0.999 .

andbeverage packaging where a greater transparency is sought.

\subsection{Adsorption Tests for Terephthalic Acid in Halloysite}

The results of the acid adsorption tests are shown in Fig. 4 terephthalic in halloysite, as well as HNT- $\mathrm{H}_{2} \mathrm{SO}_{4}$ and HNT-NaOH. This graphic shows the grams of terephthalic acid adsorbed by one gram of each type of halloysite, beingHNT- $\mathrm{H}_{2} \mathrm{SO}_{4}$ the one with the lowest amount of terephthalic acid absorbs, the aforementioned is contributed to the decrease of aluminum in the internal structure of the lumen.

\subsection{Aluminum Quantification by ICP (Inductively Coupled Plasma) in HNTs and HNTs- $\mathrm{H}_{2} \mathrm{SO}_{4}$}

In order to quantify the aluminum reduction in the HNTs with sulfuric acid treatment was quantified with the analytical technique ICP. InFig. 5 is shown the aluminum composition for the HNTs and HNT- $\mathrm{H}_{2} \mathrm{SO}_{4}$ samples where was achieved a reduction of $50 \%$ corresponding to $19 \%$ by total mass in the aluminum composition for theHNT- $\mathrm{H}_{2} \mathrm{SO}_{4}$ in comparison with the HNT not treated.

\section{Conclusion}

The aluminium change for treated HNTs was quantified with ICP technique. The aluminium reduction in HNTs was effectively achieved for $\mathrm{H}_{2} \mathrm{SO}_{4}$ treatment where is shown a reduction of $58 \% \pm$ 0.3 in the aluminum concentration with no crystalline structure modification. The halloysites treated with $\mathrm{H}_{2} \mathrm{SO}_{4}$ are more suitable to food packaging applications where aluminium concentration represents a problem.

The chemical modification with sulfuric acidfor HNTs has proven effective decrease in the HNTs diffuse reflectance without destroying their crystalline structure.

The halloysites treated with sulfuric acid have less terephthalic acid adsorption in comparison with not chemical treated HNTs because the aluminum insideinteracts with terephthalicacid. For the sodium hydroxide treatmentit does not show a difference for terephthalic acid adsorption in comparison with HNTsnot treated.

\section{Acknowledgments}

The authors thank the technical support from Dr. Eduardo Sánchez Maximianofrom UANL and Dr. Antonio Sánchez Fernández from ITESM.

\section{References}

[1] Pasbakhsh, P., Ismail, H., Fauzi, M. N. A., and Bakar, A. A.2010. "EPDM/Modified HalloysiteNanocomposites."Applied Clay Science.

[2] FHashemifard, S. A., Ismail, A. F., and Matsuura, T. 2011. "Mixed Matrix Membrane Incorporated with Large Pore Size Halloysite Nanotubes (HNT) as Filler for Gas Separation: Experimental."Journal of Colloid and Interface Science359(2): 359-70.

[3] Liu, M., Jia, Z., Jia, D., and Zhou, C. 2014. "Recent 
Advances in Research on Halloysite Nanotubes-Polymer Nanocomposite."Progress in Polymer Science39(8): 1498-525.

[4] Fujii, K., Nakagaito, A. N., Takagi, H., and Yonekura, D. 2014. "Sulfuric Acid Treatment of HalloysiteNanoclay to Improve the Mechanical Properties of PVA/Halloysite
Transparent Composite Films." Composite Interfaces 21 (4): 319-27.

[5] Monsiváis-Barrón, A. J., Bonilla-Ríos, J., and Sánchez-Fernández, A. 2014. "Property Relationship in Organosilanes and Nanotubes Filled Polypropylene Hybrid Composites.” Materials 7 (10): 7073-92. 